\title{
Redes sociais de apoio no contexto da prematuridade: perspectiva do modelo bioecológico do desenvolvimento humano
}

\author{
Social support networks in the context of prematurity: \\ Perspective of the bioecological model of \\ human development
}

\author{
Zaira Aparecida de Oliveira CUSTÓDIO \\ Maria Aparecida CREPALDI² \\ Maria Beatriz Martins LINHARES ${ }^{3}$
}

\section{Resumo}

Este artigo apresenta uma revisão crítica da literatura a respeito das redes sociais de apoio no contexto do desenvolvimento de crianças nascidas pré-termo, centrada na abordagem do Modelo Bioecológico do Desenvolvimento Humano, a qual aborda a influência de ambientes externos como contexto para o desenvolvimento. Realizou-se o levantamento bibliográfico nas bases de dados Portal da Capes, MedLine, Lilacs e SciELO, por meio de descritores pré-estabelecidos. Dentre 137 trabalhos, apenas 39 referiam-se à temática proposta, e apenas um relacionou prematuridade e modelo bioecológico. Os resultados apontam a importância do apoio de pessoas significativas e dos programas de acompanhamento de crianças que nascem pré-termo e de suas famílias, o que remete, respectivamente, à rede social pessoal e institucional. Ressalta-se a implementação das políticas de saúde que planejam as ações na saúde perinatal e na área da infância a partir da perspectiva das redes sociais de apoio como estratégia de cuidado.

Palavras-chave: Apoio social; Desenvolvimento humano; Modelo bioecológico; Nascimento prematuro.

\begin{abstract}
This article presents a critical review of the literature related to social support networks in the context of the development of children born pre-term. The focus is on the approach of the Bioecological Model of Human Development, which highlights the influence of external environments as a context for development. The bibliographical survey was carried out in the following databases, Portal da Capes, MedLine, Lilacs and SciELO, using pre-established descriptors. Among

$\because \nabla v$

1 Universidade Federal de Santa Catarina, Hospital Universitário, Maternidade. R. Profa. Maria Flora Pausenwang, Campus Universitário, 88040-970, Florianópolis, SC, Brasil. Correspondência para/Correspondence to: Z.A.O. CUSTÓDIO. E-mail: <zaira@hu.ufsc.br>.

2 Universidade Federal de Santa Catarina, Departamento de Psicologia, Programa de Pós-Graduação em Psicologia. Florianópolis, SC, Brasil.

3 Universidade de São Paulo, Faculdade de Medicina de Ribeirão Preto, Departamento de Neurociências e Ciências do Comportamento. Ribeirão Preto, SP, Brasil.
\end{abstract}


the 137 studies, only 39 referred to the proposed theme and only one related prematurity with the Bioecological Model. The results highlight the importance of support from significant people and of the monitoring programs for children born preterm and their families, referring to the personal and institutional social network, respectively. The importance of the implementation of health policies in which actions are planned in the perinatal and childhood health area is noteworthy from the perspective of social support networks as a care strategy.

Keywords: Social support; Human development; Bio-ecological model; Premature birth.

Os avanços tecnológicos que ocorreram nos últimos anos na terapia intensiva neonatal apontam para uma nova realidade: a sobrevivência significativa de recém-nascidos pré-termo e de baixo peso, especialmente os recém-nascidos de extremo baixo peso (peso inferior a 1.000g) e de muito baixo peso (peso inferior a 1.500g). O grande desafio, no entanto, está em se estabelecer como oferecer a essas crianças uma boa qualidade de vida, ao longo de seu desenvolvimento, considerando-se o alto índice de morbi-mortalidade inerente a essa condição de nascimento. Zomignani, Zambelli e Antônio (2009) ressaltam que a prematuridade se configura, a priori, como um fator de risco biológico para o desenvolvimento da criança.

Com base nessa realidade, o presente artigo pretende realizar uma revisão crítica da literatura, na perspectiva bioecológica do desenvolvimento humano, a respeito da influência das redes sociais de apoio na promoção do desenvolvimento das crianças que nascem pré-termo. Realizou-se revisão bibliográfica nas bases de dados informatizadas Portal da Capes, MedLine, Lilacs e SciELO, por meio da combinação, em conjunto, dos seguintes descritores: "prematuridade", "redes sociais de apoio", "acompanhamento de bebês pré-termo", " modelo bioecológico do desenvolvimento humano" e seus correspondentes em inglês: prematurity, social support networks, monitoring of preterm infants, bioecological model of human development. 0 período de publicação dos documentos foi delimitado entre os anos de 1980 e 2011 . Foram encontrados 137 trabalhos entre artigos publicados em periódicos científicos, teses, livros e capítulos de livro. Desses, 39 estavam relacionados à temática proposta pelo presente artigo.

A partir das buscas, nas referidas plataformas, encontrou-se apenas um estudo desenvolvido com base no Modelo Bioecológico do Desenvol- vimento Humano voltado para a temática da prematuridade. A maioria dos trabalhos encontrados aponta para os problemas de desenvolvimento a que estão submetidas as crianças nascidas pré-termo e ressalta a importância dos programas de avaliação e de intervenção. Esses trabalhos referem que os bebês que nascem pré-termo e os de baixo peso estão sujeitos a uma série de sequelas neurológicas, reinternações hospitalares e problemas de ordem emocional na infância, especialmente quando esse risco biológico (prematuridade) está associado a fatores de risco ambiental, como socioeconômicos, psicossociais e de falta de estrutura familiar (Laucht, Esser, \& Schmidt, 1997; Silva, 2002).

\section{O estudo da prematuridade na perspectiva bioecológica}

Abordar a prematuridade a partir do paradigma das redes significa concebê-la como um processo complexo que envolve diversos aspectos: os biológicos, próprios de cada recém-nascido, e os referentes aos diversos ambientes dos quais ele participa; os tecnológicos, do serviço de saúde, e das intervenções da equipe multidisciplinar; além dos aspectos referentes à relação dos pais e/ou cuidadores dessa criança.

Nesse sentido, é imprescindível a utilização de modelos teóricos que não dissociem o sujeito dos múltiplos contextos nos quais ele concretiza seu desenvolvimento. O Modelo Bioecológico do Desenvolvimento Humano, proposto por Bronfenbrenner (1996; 2005), é uma abordagem teórico-metodológica que abarca essa concepção, pois prioriza o estudo do desenvolvimento humano no seu contexto de vida real, vislumbrando todo o sistema ecológico no qual ele ocorre, bem como as interações nele estabelecidas (Kazak, Segal-Andrews, \& Johnson, 1995). 
Falar em desenvolvimento humano implica considerar que ele ocorre contextualmente com base nos quatro componentes dinâmicos e inter-relacionados propostos por Bronfenbrenner: a Pessoa, o Processo, o Contexto e o Tempo - modelo PPCT (Bronfenbrenner, 1996; Bronfenbrenner \& Morris, 1998; Bronfenbrenner \& Evans, 2000). A Pessoa é compreendida a partir de suas características biopsicológicas e daquelas construídas na interação com o ambiente. O Processo é visto como o responsável pelo desenvolvimento e se dá por meio da interação recíproca e cada vez mais complexa entre um ser humano e outras pessoas, objetos e símbolos em um ambiente imediato, que Bronfenbrenner chamou de processos proximais. 0 Contexto refere-se aos contextos de vida da pessoa e é analisado por meio da interação de quatro níveis ambientais: o microssistema, o mesossistema, o exossistema e o macrossistema, e todos esses sistemas veem-se influenciados pelo cronossistema. Esses sistemas, de acordo com Bronfenbrenner (1996), estão organizados como um encaixe de estruturas concêntricas, compondo o que esse autor chama de meio-ambiente-ecológico.

O microssistema é o ambiente imediato no qual está a pessoa em desenvolvimento a Unidade de Terapia Intensiva (UTI) - Neonatal e a família, por exemplo), e no qual se estabelecem relações face a face estáveis, com reciprocidade e afeto. É no microssistema que ocorrem os processos proximais que produzem e sustentam o desenvolvimento e cuja eficácia depende da sua estrutura e do seu conteúdo (Bronfenbrenner \& Morris, 1998). O mesossistema diz respeito às inter-relações entre dois ou mais ambientes (microssistemas) de que a pessoa em desenvolvimento participa, como as relações entre a família e a creche, a família e o trabalho, entre outros.

No exossistema, por sua vez, a pessoa não se encontra presente, mas as relações que nele existem afetam indiretamente seu desenvolvimento, como trabalho dos pais, rede social de apoio e comunidade. Segundo Kazak et al. (1995), "pesquisa nesta área considerou o impacto das redes parentais e do trabalho dos pais sobre as crianças" (p.87). O macrossistema consiste nos valores, nas políticas, na cultura, nos modelos de atuação da equipe de saúde e nos obstáculos no curso da vida que influenciam o desenvolvimento das pessoas (Bronfenbrenner \& Morris, 1998).

Para Bronfenbrenner (1996), as relações ocorridas nos subsistemas, e entre eles, podem ser mais decisivas para o desenvolvimento humano do que os próprios eventos que ocorrem em cada um deles. Assim, pode-se inferir que a promoção de saúde e o desenvolvimento de crianças nascidas pré-termo vão depender, além do engajamento da família e dos serviços de saúde, sobretudo, do tipo de interação estabelecida entre ambos.

O Tempo, quarto componente do modelo PPCT, permite examinar a influência de mudanças e continuidades para o desenvolvimento humano que ocorrem ao longo do ciclo de vida. Nesse sentido, o cronossistema diz respeito ao tempo, pela transformação que ocorre nos subsistemas ao longo do tempo, considerando a história transgeracional da família e dos grupos sociais (Polônia, Dessen, \& Silva, 2005).

As redes sociais de apoio estão inscritas no componente Contexto, no qual é possível vislumbrar as interações envolvidas no desenvolvimento, como o das crianças nascidas pré-termo. Para Zucchi (1999), a prematuridade é um tema preocupante não só pelos índices de mortalidade a ela associados, mas pela qualidade de vida restrita aos que a ela sobrevivem. Embora tenha havido avanços nas últimas décadas, com recursos humanos e tecnologias complexas que permitem a sobrevivência do recém-nascido de extremo baixo peso, as sequelas da prematuridade podem ser graves (Rugolo, 2005). Os problemas estão relacionados, principalmente, ao desenvolvimento cognitivo e comportamental (Espírito Santo, Portuguez, \& Nunes, 2009), motor (Halpern, Giugliani, Victora, Barros, \& Horta, 2000) e de linguagem (Custódio, 2010; Nobre, Carvalho, Martinez, \& Linhares, 2009).

Outro aspecto inerente ao recém-nascido pré-termo são as intercorrências clínicas da sua condição neonatal, que, conforme Fraga, Linhares, Carvalho e Martinez (2008), podem ser: as doenças respiratórias, apneias, anemia, displasia broncopulmonar, retinopatia da prematuridade, hemorragia 
intracraniana, pneumotórax, entre outros. A condição neonatal, avaliada nas primeiras horas após o nascimento, juntamente com a evolução clínica do bebê indicarão o prognóstico do desenvolvimento da criança (Linhares et al., 2004).

A partir da alta dos recém-nascidos pré-termo, surgem questões relevantes sobre a trajetória de seu desenvolvimento. Esses bebês estão sujeitos a uma maior incidência de problemas na infância, pois, além da prematuridade, normalmente estão expostos a outros fatores de risco para o seu desenvolvimento. Nesse sentido, compreender a função da rede social de apoio no contexto da prematuridade permite vislumbrar os diversos ambientes em que os bebês e suas famílias estão envolvidos, bem como os recursos disponíveis. Desse modo, possibilita-se a identificação dos fatores de risco e de proteção presentes nesses ambientes, visando ao planejamento de intervenções em saúde que contribuam para a promoção do desenvolvimento dessas crianças.

\section{Redes sociais de apoio}

Pensar uma sociedade sob a perspectiva das redes é concebê-la na sua mais ampla interdependência, o que vai ao encontro do Modelo Bioecológico, que postula as interconexões entre os diversos ambientes. A rede social é a melhor metáfora que representa esse modelo, pois reconhece a interação entre diferentes setores e suas práticas e permite a reflexão sobre a cultura na qual se está imerso (Dabas, 2000).

As redes sociais são definidas por Sluzki (1997) como as relações que compreendem não apenas a família nuclear ou extensa, mas os vínculos interpessoais ampliados, como os amigos, os colegas de trabalho ou de estudo e as relações que são estabelecidas na comunidade. Esse tipo de rede proporciona o apoio social que remete à ajuda mútua potencializada quando uma rede social é forte e integrada.

Os primeiros trabalhos sobre redes foram desenvolvidos na década de 1960, nos Estados Unidos, em comunidades menos privilegiadas na área de saúde mental, pois o problema que as pessoas viviam não podia ser dissociado do contexto mais amplo das suas relações (Sluzki, 1997). A partir da década de 1990, as redes sociais e o apoio social ocupam o centro das pesquisas em ciências sociais internacionalmente. Destacam-se autores como Dabas (1993), Klefbeck (2000), Sluski (1997), entre outros, que desenvolvem a temática das redes pessoais e sociais em diferentes contextos. Para Klefbeck (2000), as redes sociais vêm-se tornando foco de muitos estudos devido a sua importância comprovada no bem-estar e nas situações de crise. Esses estudos têm como precursor o Modelo Bioecológico do Desenvolvimento.

Adotar a perspectiva de redes no campo da saúde permite elaborar uma intervenção com papéis e funções definidas, além de identificar as interações estabelecidas e o modo como as pessoas lidam com a experiência da doença. Serapioni (2005) indica que, nos últimos vinte e cinco anos, pesquisas têm destacado o papel fundamental da família e das redes sociais no que se refere à atenção e à promoção da saúde. Nessa perspectiva, Sluzki (1997) correlaciona a saúde das pessoas com o estabelecimento de uma rede social estável, ativa e confiável. Para Sluzki (1997), a carência relativa de relações sociais é considerada como um fator de risco.

O apoio social é fundamental ao longo do desenvolvimento humano, de acordo com Dessen e Braz (2000), e tem destaque durante períodos de transição e de mudanças. O nascimento de um filho é uma das situações em que se requer adaptação, pois inclui, por exemplo, as demandas do bebê e as mudanças na interação conjugal. O nascimento de um bebê pré-termo e sua internação numa UTI neonatal configuram-se, especialmente, uma situação de crise, com repercussões sobre todo o grupo familiar que podem comprometer o estabelecimento dos vínculos afetivos e os cuidados destinados ao bebê, bem como a trajetória de seu desenvolvimento (Custódio, 2010; Linhares, 2002). A equipe de saúde da UTI neonatal deve ser capaz de perceber as tramas implícitas e explícitas, nesse contexto, a fim de identificar com a família os caminhos na construção de redes sociais de apoio que sejam efetivas. 
No contexto da saúde perinatal, diante de uma situação de crise e de riscos impostos pela prematuridade, é necessário conhecer os recursos pessoais e institucionais de que a família dispõe. Segundo Pinelli (2000), o ajustamento familiar ou habilidade para realizar mudanças no sistema familiar, a partir de um evento estressor, como a prematuridade, está relacionado aos recursos internos e sociais da família e às estratégias de enfrentamento utilizados.

Quando se aborda a questão das redes no contexto da saúde, segundo Dabas (2000), considera-se que sua aplicação ultrapassa as instituições que oferecem esses serviços, como hospital, unidades de saúde etc. Essa autora distingue os seguintes níveis de rede em relação à saúde: rede pessoal, rede institucional, rede comunitária, rede de serviços e rede intersetorial. Deve-se considerar, no entanto, que essas divisões são arbitrárias, pois pode ocorrer, entre elas, um intercâmbio dinâmico. Este artigo, no entanto, focará a rede pessoal e institucional no contexto do desenvolvimento de crianças nascidas pré-termo.

\section{Rede social pessoal}

Dentre os vários ambientes que sustentam e fazem parte do universo de relações do indivíduo, está no nível de seu microssistema a rede social pessoal, definida por Sluzki (1997, p.42) como: "a soma de todas as relações que um indivíduo percebe como significativas ou define como diferenciadas da massa anônima da sociedade". Essas redes, como sugere o termo, funcionam como pontos de apoio e contribuem para a experiência individual de identidade e de competência, na qual se incluem os hábitos de cuidado à saúde e a adaptação nos momentos de crise.

A rede social pessoal tem-se mostrado benéfica para o comportamento e para as emoções da nova mãe, em um momento gerador de inseguranças devido às aceleradas transformações pelas quais ela passa (Custódio, 2010; Simioni \& Geib, 2008). Esses autores ressaltam que as mães com uma rede social mais consistente, e que podem, portanto, ter suas necessidades emocionais atendi- das, tendem a solicitar mais apoio para elas mesmas, bem como a manter uma interação mais sensível com o bebê.

A mãe de bebê pré-termo apresenta uma necessidade particular de acolhimento para sua dor e sofrimento intensos. Ela precisa ser reassegurada em relação aos cuidados que desempenha diante de seu filho, que necessita de cuidados especializados. A vivência psíquica materna é intensa: desejo, culpa, medo e ansiedade se misturam, fazendo com que se sinta isolada dentro do seu sofrimento (Brito \& Pessoa, 2006; Linhares, Carvalho, Bordin, \& Jorge, 1999; Linhares et al., 2004).

Diante de tal situação, a mãe necessita de uma rede cuja função seja a de apoio emocional, que, conforme Sluzki (1997), consiste numa relação de empatia, estímulo e apoio estabelecidos através de amizades íntimas e de relações familiares significativas. Em seu estudo, Custódio (2010) constatou, por meio do diagrama do Ecomapa, que o apoio emocional foi o predominante na rede social da mãe ao longo dos dois primeiros anos de vida da criança nascida pré-termo. Nesse estudo, o pai foi quem mais ofereceu esse tipo de apoio, seguido da avó materna e da equipe de saúde da UTI Neonatal. Muitas vezes, para poder acompanhar o bebê internado, a mãe precisa de pessoas que desempenhem outras funções de apoio, como a de ajuda prática em casa com os outros filhos. Da mesma forma, a mãe precisa de pessoas que possam compartilhar informações e experiências pessoais ou sociais, servindo, assim, como guia cognitivo.

Stern (1997) refere-se a esse tipo de apoio ofertado à mãe como uma "rede maternal", pois tem a função de "protegê-la fisicamente, prover suas necessidades vitais, afastá-la da realidade externa para que ela possa ocupar-se de seu bebê" (p.31). Nessas condições, a mulher será capaz de alcançar bons resultados nas tarefas de manter 0 bebê vivo, crescendo e se desenvolvendo fisicamente, além de envolver-se emocionalmente com ele, amando-o, identificando-se sensivelmente para melhor responder às suas necessidades (Silva, 2003; Stern, 1997).

Muller-Nix et al. (2004) assinalam a necessidade de se detectarem pais com experiências 
estressantes na situação de prematuridade a fim de planejar intervenções de apoio no período neonatal, o que poderá ajudá-los a lidar com as dificuldades da transição para a parentalidade. Prover suporte emocional e social para os pais pode moderar o efeito adverso dessa experiência, bem como ter um efeito positivo na interação entre os pais e a criança. De acordo com esses autores, o impacto da qualidade dessa interação é muito importante. Os avanços nesse sentido, no entanto, datam somente de duas décadas, com a presença dos pais na unidade neonatal e o aumento do suporte emocional oferecido a eles nesse período.

Após a fase crítica da internação na unidade neonatal e com a alta hospitalar, a mãe e os demais familiares levam para casa um bebê que requer cuidados especiais e com poucos recursos de interação, conforme ressaltam Linhares et al. (1999). É fundamental que o bebê que nasceu pré-termo seja acolhido em um microssistema que promova e proteja seu desenvolvimento. Para tanto, a equipe de saúde deve identificar quem compõe a rede social dos pais e com quem eles podem contar. Segundo Klefbeck (2000), é fundamental a equipe de saúde avaliar o grau de dificuldade encontrado pela família. Assim, verifica-se a necessidade de ser acionada uma rede de apoio que possibilite à família acompanhar o desenvolvimento da criança juntamente com outros serviços de saúde.

\section{Rede social institucional}

A rede social institucional diz respeito à rede de apoio oferecida por meio dos serviços de saúde disponíveis na instituição hospitalar quando do nascimento do bebê pré-termo e, posteriormente, pelos serviços de acompanhamento ambulatorial, como o Ambulatório de Seguimento (follow-up) e/ou a Unidade Básica de Saúde (UBS). O objetivo dessa rede seria fornecer apoio técnico e integral aos bebês e suas famílias de forma a satisfazer às suas necessidades individuais de saúde, protegê-los dos riscos e promover o seu desenvolvimento.

No hospital, a unidade neonatal é o primeiro microssistema do bebê, composto por profissionais

252 de diversas áreas da saúde (Lamy Filho, 2003). Essa é a primeira rede social institucional que o bebê e a família dispõem para atender suas demandas de saúde. As unidades neonatais no Brasil vêm trabalhando um modelo de assistência conhecido como Atenção Humanizada ao Recém-Nascido de Baixo Peso - Método Canguru (MC). Esse método, centrado na humanização, compreende ações voltadas para o desenvolvimento da criança desde o pré-natal de alto risco, passando pela unidade neonatal, até a alta hospitalar do bebê (Brasil, 2011). O MC é desenvolvido em três etapas: a $1^{\text {a }}$ e a $2^{\mathrm{a}}$ no âmbito hospitalar, e a $3^{\text {a }}$, no ambulatorial, cujos fundamentos básicos são norteados pelo respeito à subjetividade e à capacidade neurológica do bebê e pelo acolhimento de sua família.

A alta hospitalar passa a ser um marco no desenvolvimento do bebê, que requer um acompanhamento sistemático. Linhares et al. (2004) ressaltam a importância da intervenção preventiva do follow-up como uma estratégia de proteção ao desenvolvimento da criança nascida pré-termo e que busca desencadear ou ativar o processo de resiliência nesse grupo, que se configura com potencial de risco de transtornos de desenvolvimento.

Há consenso quanto à importância do acompanhamento diferenciado a essas crianças, e a Organização Mundial de Saúde estabeleceu protocolos para a realização desse acompanhamento (Kumar et al., 2008). Recomenda-se que os serviços organizem um programa com retornos regulares aos 3 , $6,9,12,18$ e 24 meses pelo menos, com uma avaliação psicossocial (Brasil, 2011). Contudo, ainda é discutido até que idade deve haver esse acompanhamento, pois os problemas de desenvolvimento raramente são identificados nos primeiros anos de vida (Dorling \& Field, 2006). Segundo Linhares et al. (2000), os programas de intervenção devem acompanhar a criança até que ela complete seis ou sete anos de idade, quando tem início o desafio do ensino fundamental.

Entende-se que quanto maior for o tempo de acompanhamento da criança no follow-up, mais detalhada será a avaliação das consequências dos fatores de risco no funcionamento neuropsicológico, cognitivo e na coordenação motora mais complexa (Salt \& Redshaw, 2006). Um dos objetivos do 
follow-up é dar suporte à família, por meio de esclarecimentos e de apoio, facilitando seu vínculo com os membros da equipe a fim de reduzir os danos de desenvolvimento na criança.

No estudo de Custódio (2010), foi verificado que as crianças com classificação de risco para o desenvolvimento, avaliadas pelo Teste de Denver II, apresentaram riscos associados, como famílias com índice de risco psicossocial de Rutter severo e moderado, ambientes com baixa responsividade e envolvimento emocional, rede social de apoio composta por poucos membros, falta de conexão entre os microssistemas e escassez de apoio pessoal e institucional. Entretanto, o referido estudo mostrou que as crianças com resultado normal no Teste de Denver II apresentaram baixo risco psicossocial de Rutter e fatores de proteção na rede de apoio das famílias, como tamanho médio da rede, conexão entre os microssistemas e desempenho de diversas funções de apoio pelos membros da rede.

Segundo Serapioni (2005), atualmente, busca-se, nacional e internacionalmente, retomar a família como unidade de atenção das políticas públicas, desenvolver redes de apoio e de envolvimento das famílias e comunidades, além de realizar uma melhor integração entre famílias, serviços públicos e iniciativa do setor informal, o que vai ao encontro do modelo bioecológico. Nessa perspectiva, Bronfenbrenner e Evans (2000) analisaram a influência de ambientes externos sobre o funcionamento das famílias e sua capacidade em promover o desenvolvimento saudável de seus filhos. Esses autores sugerem a organização de estratégias de intervenção direcionadas aos ambientes promotores de saúde como contexto para o desenvolvimento humano.

Considerando que muitos bebês pré-termo são encaminhados para a UBS, a comunicação entre os diferentes níveis de atenção na organização desses serviços seria uma forma de otimizar recursos, ressalta Meio, Magluta, Mello e Moreira (2005). Em seus achados, Custódio (2010) constatou que a vigilância do desenvolvimento na UBS não é realizada de forma satisfatória, além da presença de outros fatores de risco, como o tipo de interação com a família, que é marcada por dificuldades e distanciamento. As dificuldades apresentadas pelas famílias neste microssistema foram: acesso e agendamento às consultas para as crianças e familiares, deficiências de conhecimento sobre o desenvolvimento infantil por parte de alguns profissionais, inexistência do pediatra e trocas constantes de profissionais na unidade, demora nos encaminhamentos para a realização de exames, consultas especializadas e cirurgias.

Para os profissionais de saúde atuarem com uma abordagem familiar e integrada, Dabas (2000) sugere que, além da sua capacitação técnica, seja incluída a importância da rede social de apoio. Além de mapear essa rede, é necessário concebê-la como uma ação estratégica na promoção da saúde e na redução dos danos para o desenvolvimento. No acompanhamento do desenvolvimento da criança, deve-se considerá-la, juntamente com sua família, como participante do micro e do macrossistema, com os quais interage de maneira constante (Nikodem, 2009).

\section{Considerações Finais}

A abordagem de rede no contexto da prematuridade, sob a concepção do Modelo Bioecológico do Desenvolvimento Humano, permite vislumbrar a complexidade dos vários sistemas envolvidos nesse cenário, além da inter-relação entre eles. O intercâmbio contínuo e recíproco entre a família e sua rede social de apoio, além de outros tecidos que compõem a sociedade civil, fortalece as ações em rede na área da saúde. Assim, espera-se um futuro mais promissor em relação ao acompanhamento do desenvolvimento das crianças nascidas pré-termo. Por meio da conexão dinâmica dos vários pontos que formam a rede de apoio, buscar-se-á atender às necessidades de saúde das crianças e oferecer suporte às famílias.

Considerando-se os riscos para o desenvolvimento da criança no contexto da prematuridade, é fundamental a identificação da rede de apoio institucional durante a internação na unidade neonatal e após a alta hospitalar. Espera-se dessas redes o suporte técnico e integral aos bebês e suas famílias a fim de protegê-los dos riscos e promover seu desenvolvimento. Da mesma forma, é fundamental conhecer a rede pessoal da criança e da família, na 
qual se encontram presentes as pessoas que the são significativas.

Conforme os autores pesquisados, o apoio social advindo das redes é especialmente fundamental durante os períodos de transição e de mudanças, como o nascimento de um filho pré-termo. Constata-se a importância de a organização de programas de intervenção e de acompanhamento a essas crianças e suas famílias incluírem esse tipo de apoio. Na mesma direção, é imprescindível a implementação das políticas de saúde que planejam as ações na saúde perinatal e na área da infância a partir da concepção das redes, como estratégia e política de cuidado.

Todo o investimento técnico, científico e financeiro direcionado às crianças nascidas pré-termo durante a internação neonatal só terá valor se elas forem acompanhadas em contextos que promovam seu desenvolvimento. Nessa direção, os autores pesquisados sugerem que se busque a proteção destas crianças por meio de medidas que envolvam suas famílias e os demais ambientes nos quais estão inseridas desde o micro ao macrossistema.

Ressalta-se a importância de estudos acerca dos recursos no âmbito pessoal e institucional, nos quais se encontram as redes sociais de apoio. Recomenda-se também estudos que visem investigar o microssitema e o mesossistema das crianças nascidas pré-termo e de suas famílias, especialmente as conexões existentes entre elas, uma vez que essas conexões favorecem a efetividade da rede.

Sugere-se, ainda, o estudo dos microssistemas institucionais que operam na saúde e no desenvolvimento da criança, ou seja, os serviços de saúde. Devem ser valorizadas igualmente pesquisas que abordem os aspectos do macrossistema voltados às políticas que planejam as ações de saúde, na perspectiva da produção e do fortalecimento das redes de apoio na área da criança e nas intervenções que ofereçam suporte à família.

\section{Referências}

Brasil. Ministério da Saúde. (2011). Atenção humanizada ao recém-nascido de baixo peso: Método Canguru.
Brito, M. H. A., \& Pessoa, V. L. M. P. (2006). Um perfil da mãe prematura. In R. G. Melgaço (Org.), A ética na atenção ao bebê: psicanálise-saúde-educação (pp.115-124). São Paulo: Casa do Psicólogo.

Bronfenbrenner, U. (1996). A ecologia do desenvolvimento humano: experimentos naturais e planejados. Porto Alegre: Artes Médicas.

Bronfenbrenner, U. (2005). Making humans beings human: Biological perspectives on human development. London: Sage.

Bronfenbrenner, U., \& Morris, P. (1998). The ecology of developmental processes. In W. Damon (Org.), Handbook of child psychology (Vol. 1, pp.993-1027). New York: John Wiley \& Sons.

Bronfenbrenner, U., \& Evans, G. (2000). Developmental science in the $21^{\text {st }}$ century: Emerging questions, theoretical models, research designs and empirical findings. Social Development, 9(1), 115-125.

Custódio, Z. A . O. (2010). Redes sociais no contexto da prematuridade: fatores de risco e de proteção para o desenvolvimento da criança ao longo de seus dois primeiros anos de vida (Tese de doutorado não-publicada). Universidade Federal de Santa Catarina, Florianópolis.

Dabas, E. (1993). Red de redes: lás prácticas de intervención em redes sociales. Buenos Aires: Paidos.

Dabas, E. (2000). Comentário de Elina Dabas. Revista Sistemas Familiares, 16(1), 57-63.

Dessen, M. A., \& Braz, M. P. (2000). Rede social de apoio durante transições familiares decorrentes do nascimento de filhos. Psicologia: Teoria e Pesquisa, 16(3), 221-231.

Dorling, J. S., \& Field, D. J. (2006). Follow up of infants following discharge from the neonatal unit: Structure and process. Early Human Development, 82(3), 151-156.

Espírito Santo, J. L., Portuguez, M. W., \& Nunes, M. L. (2009). Status cognitivo comportamental de prematuros de baixo peso ao nascer em idade pré-escolar que vivem em país em desenvolvimento. Jornal de Pediatria, 85(1), 35-41.

Fraga, D. A., Linhares, M. B. M., Carvalho, A. E. V., \& Martinez, F. E. (2008). Desenvolvimento de bebês prematuros relacionado a variáveis neonatais e maternas. Psicologia em Estudo, 13(2), 335-344.

Halpern, R., Giugliani, E. R. J., Victora, C. G., Barros, F. C., \& Horta, B. L. (2000). Fatores de risco para suspeita de atraso no desenvolvimento neuropsicomotor aos 12 meses de vida. Jornal de Pediatria, 76(6), 421-428.

Kazak, A. E., Segal-Andrews, A. M., \& Johnson, K. (1995). Pediatric psychology research and practice: A family/ systems approach. In M. C. Roberto (Ed.), Handbook of pediatric psychology ( $2^{\text {nd }}$ ed., pp.88-104). London: The Guilford Press,

Klefbeck, J. (2000). Terapia de red: un método de tratamiento en situaciones de crisis. Revista Sistemas Familiares, 16(1), 47-56. 
Kumar, P., Sankar, M. J., Sapra, S., Agarwal, R., Deorari, A., \& Paul, P. (2008). Follow-up of high risk neonates. WHO collaborating centre for training and research in newborn care. Retrieved December 15, 2009, from http://www.newbornwhocc.org/pdf/Follow-up_of_ High_Risk_Neonates_050 508.pdf

Lamy Filho, F. (2003). A equipe da UTI neonatal. In M. E. L., Moreira, N. A. Braga \& D. S. Morsch (Org.), Quando a vida começa diferente: o bebê e sua familia na UTI neonatal (pp.107-166). Rio de Janeiro: Editora Fio Cruz.

Laucht, M., Esser, G., \& Schmidt, M. H. (1997). Developmental outcome of infants born with biological and psychosocial risks. Journal Child Psychology and Psychiatry, 38(7), 843-853.

Linhares, M. B. M. (2002). Prematuridade, risco e mecanismos de proteção do desenvolvimento. Temas Sobre Desenvolvimento, 12(Supl. Esp.), 18-24.

Linhares, M. B. M., Carvalho, A. E. V., Bordin, M. B. M., Chumello, J. T., Martinez, F. E., \& Jorge, S. M. (2000). Prematuridade e muito baixo peso como fatores de risco ao desenvolvimento da criança. Paidéia, 10(18), 60-69.

Linhares, M. B. M., Carvalho, A. E. V., Bordin, M. B. M., \& Jorge, S. M. (1999). Suporte psicológico ao desenvolvimento de bebês pré-termo com peso de nascimento <1500g: na UTI-neonatal e no segmento longitudinal. Temas em Psicologia, 7(3), 245-262.

Linhares, M. B. M., Carvalho, A. E. V., Padovani, F. H. P., Bordin, M. B. M., Martins, I. M. B., \& Martinez, F. E. (2004). A compreensão do fator de risco da prematuridade sob a ótica desenvolvimental. In E. M. Marturano, M. B. M. Linhares \& S. R. Loureiro (Orgs.), Vulnerabilidade e proteção: indicadores na trajetória de desenvolvimento do escolar (pp.75-106). São Paulo: Casa do Psicólogo.

Meio, M. D. B. B., Magluta, C., Mello, R. R., \& Moreira, M. E. L. (2005). Análise situacional do atendimento ambulatorial prestado a recém-nascidos egressos das unidades de terapia intensiva neonatais no estado do Rio de Janeiro. Ciência \& Saúde Coletiva, 10(2), 299-307.

Muller-Nix, C., Forcada-Guex, M., Perrehumbert, B., Jaunin, L., Borghini, A., \& Ansermet, F. (2004). Prematurity, maternal stress and mother-child interactios. Early Human Development, 79,145-158.

Nobre, F. D. A., Carvalho, A. E. V., Martinez, F. E., \& Linhares, M. B. M. (2009). Estudo longitudinal do desenvolvimento de crianças nascidas pré-termo no primeiro ano pósnatal. Psicologia: Reflexão e Crítica, 22(3), 362-369.
Nikodem, M. R. (2009). Niños de alto riesgo: intervenciones tempranas en el desarrollo y la salud infantil. Buenos Aires: Paidós.

Pinelli, J. (2000). Effects of family coping and resources on family adjustment and parental stress in the acure phase of the NICU experience. Neonatal Newtork, 19(6), 27-37.

Polônia, A. C., Dessen, M. A., \& Silva, N. L. P. (2005). O modelo bioecológico de Bronfenbrenner: contribuições para o desenvolvimento humano. In M. A. Dessen \& A. L. Costa Junior (Orgs.), A ciência do desenvolvimento humano: tendências atuais e perspectivas futuras (pp.19-36). Porto Alegre: Artmed.

Rugolo, L. M. (2005). Crescimento e desenvolvimento a longo prazo do prematuro extremo. Jornal da Pediatria, 81(1 Supl.), S101-S110.

Salt, A., \& Redshaw, M. (2006). Neurodevelopmental follow-up after preterm birth: Follow-up after two years. Early Human Development, 8(3), 185-197.

Serapioni, M. (2005). O papel da família e das redes primárias na reestruturação das políticas sociais. Ciência \& Saúde Coletiva, 10(Supl.), 243-253.

Silva, M. R. S. (2003). A construção de uma trajetória resiliente durante as primeiras etapas do desenvolvimento da criança: o papel da sensibilidade materna e do suporte social (Tese de doutorado não-publicada). Universidade Federal de Santa Catarina, Florianópolis.

Silva, O. P. V. (2002). A importância da família no desenvolvimento do bebê prematuro. Psicologia: Teoria e Prática, 4(2)15-24.

Simioni, A. S., \& Geib, L. T. C. (2008). Percepção materna quanto ao apoio social recebido no cuidado às crianças prematuras no domicílio. Revista Brasileira de Enfermagem, 61(5), 645-51.

Sluzki, C. A. (1997). A rede social na prática sistêmica: alternativas terapêuticas. São Paulo: Casa do Psicólogo.

Stern, D. N. (1997). A constelação da maternidade. Porto Alegre: Artes Médicas.

Zomignani, A. P., Zambelli, H. J. L., \& Antonio, M. A. R. G. M. (2009). Desenvolvimento cerebral em recémnascidos prematuros. Revista Paulista Pediatria, 27(2), 198-203.

Zucchi, M. (1999). Depressão na gravidez e prematuridade: aspectos epistemológicos da investigação. Cadernos de Saúde Pública, 15(1), 89-97.

Recebido em: 2/2/2012

Versão final em: 5/6/2012

Aprovado em: 19/6/2012 
